\title{
MULTIPLIERS AND IMAGINARY POWERS OF THE SCHRÖDINGER OPERATORS CHARACTERIZING UMD BANACH SPACES
}

\author{
Jorge J. Betancor, Raquel Crescimbeni, \\ Juan C. Fariña and Lourdes Rodríguez-Mesa
}

\begin{abstract}
Universidad de la Laguna, Departamento de Análisis Matemático Campus de Anchieta, Avda. Astrofísico Francisco Sánchez, s/n 38271 La Laguna, (Sta. Cruz de Tenerife), España; jbetanco@ull.es Universidad Nacional de Comahue, Departamento de Matemáticas 8300 Neuquén, Argentina; raquel.crescimbeni@faea.uncoma.edu.ar Universidad de la Laguna, Departamento de Análisis Matemático Campus de Anchieta, Avda. Astrofísico Francisco Sánchez, s/n 38271 La Laguna (Sta. Cruz de Tenerife), España; jcfarina@ull.es Universidad de la Laguna, Departamento de Análisis Matemático Campus de Anchieta, Avda. Astrofísico Francisco Sánchez, s/n 38271 La Laguna (Sta. Cruz de Tenerife), España; lrguez@ull.es
\end{abstract}

\begin{abstract}
In this paper we establish $L^{p}$-boundedness properties for Laplace type transform spectral multipliers associated with the Schrödinger operator $\mathcal{L}=-\Delta+V$. We obtain for this type of multipliers pointwise representation as principal value integral operators. We also characterize the UMD Banach spaces in terms of the $L^{p}$-boundedness of the imaginary powers $\mathcal{L}^{i \gamma}, \gamma \in \mathbf{R}$, of $\mathcal{L}$.
\end{abstract}

\section{Introduction}

We study certain class of spectral multipliers, usually called Laplace transform type multipliers associated with the Schrödinger operator $\mathcal{L}=-\Delta+V$, where $\Delta$ represents the Laplacian operator and the potential $V>0$ satisfies a reverse Hölder inequality. We prove that $L^{p}$-boundedness of some of those multipliers, the imaginary powers of $\mathcal{L}$, acting on Banach valued functions characterizes the UMD property for the Banach space.

We now recall some definitions and properties that will be useful in order to state and to prove our results.

We consider the Schrödinger operator $\mathcal{L}=-\Delta+V$ on $\mathbf{R}^{n}$, with $n \geq 3$. We assume that $V>0$ is a locally integrable function on $\mathbf{R}^{n}$ belonging to the class $B_{q}$, that is, there exists $C>0$ such that, for every ball $B$ in $\mathbf{R}^{n}$,

$$
\left(\frac{1}{|B|} \int_{B} V^{q}(x) d x\right)^{1 / q} \leq C \frac{1}{|B|} \int_{B} V(x) d x
$$

for some $q \geq n / 2$.

doi:10.5186/aasfm.2013.3813

2010 Mathematics Subject Classification: Primary 42C05; Secondary 42C15.

Key words: UMD spaces, Schrödinger operators, multipliers, imaginary powers.

The first, third and fourth author are partially supported by MTM2010-17974. The second author is partially supported by the Universidad Nacional de Comahue. 
The operator $\mathcal{L}$, suitably understood (see, for instance, [2]), is a closed unbounded and positive operator in $L^{2}\left(\mathbf{R}^{n}\right)$. Then, there exists the spectral measure $E_{\mathcal{L}}$ associated with $\mathcal{L}$. It is not hard to see that 0 is not an eigenvalue of $\mathcal{L}$ in $L^{2}\left(\mathbf{R}^{n}\right)$, and hence $E_{\mathcal{L}}(\{0\})=0$. For every Borel measurable bounded function $m$ on $[0, \infty)$, we define the spectral multiplier $T_{m}^{\mathcal{L}}$ by

$$
T_{m}^{\mathcal{L}}(f)=\int_{(0, \infty)} m(\lambda) E_{\mathcal{L}}(d \lambda) f, \quad f \in L^{2}\left(\mathbf{R}^{n}\right) .
$$

It is well known that $T_{m}^{\mathcal{L}}$ defines a bounded operator from $L^{2}\left(\mathbf{R}^{n}\right)$ into itself.

We say that a function $m$ on $(0, \infty)$ is of Laplace transform type when $m(\lambda)=$ $\lambda \int_{0}^{\infty} e^{-\lambda t} \phi(t) d t, \lambda \in(0, \infty)$, for a certain $\phi \in L^{\infty}(0, \infty)$. Note that in this case $m$ is continuous and bounded on $(0, \infty)$. The spectral multiplier $T_{m}^{\mathcal{L}}$ is called of Laplace transform type when the function $m$ is of Laplace transform type.

For every $t>0$, we define the operator $W_{t}^{\mathcal{L}}$ by

$$
W_{t}^{\mathcal{L}}(f)=\int_{(0, \infty)} e^{-\lambda t} E_{\mathcal{L}}(d \lambda) f, \quad f \in L^{2}\left(\mathbf{R}^{n}\right) .
$$

The uniparametric family $\left\{W_{t}^{\mathcal{L}}\right\}_{t>0}$ is the semigroup of operators generated by $-\mathcal{L}$ in $L^{2}\left(\mathbf{R}^{n}\right)$. For every $t>0$ and $1 \leq p \leq \infty$ the operator $W_{t}^{\mathcal{L}}(f)$ can be extended from $L^{p}\left(\mathbf{R}^{n}\right) \cap L^{2}\left(\mathbf{R}^{n}\right)$ to $L^{p}\left(\mathbf{R}^{n}\right)$ as a contraction from $L^{p}\left(\mathbf{R}^{n}\right)$ into itself. The semigroup of operators $\left\{W_{t}^{\mathcal{L}}\right\}_{t>0}$ is not conservative. Moreover, we can write, for every $t>0$ and $f \in L^{p}\left(\mathbf{R}^{n}\right), 1 \leq p \leq \infty$,

$$
W_{t}^{\mathcal{L}}(f)(x)=\int_{\mathbf{R}^{n}} W_{t}^{\mathcal{L}}(x, y) f(y) d y, \quad x \in \mathbf{R}^{n},
$$

where $W_{t}^{\mathcal{L}}(x, y), x, y \in \mathbf{R}^{n}$ and $t \in(0, \infty)$, is a $C^{\infty}\left(\mathbf{R}^{n} \times \mathbf{R}^{n} \times(0, \infty)\right)$ such that, according to Feynman-Kac property,

$$
\left|W_{t}^{\mathcal{L}}(x, y)\right| \leq C W_{t}(x, y), \quad x, y \in \mathbf{R}^{n} \text { and } t>0,
$$

being

$$
W_{t}(x, y)=\frac{1}{(4 \pi t)^{n / 2}} e^{-\frac{|x-y|^{2}}{4 t}}, \quad x, y \in \mathbf{R}^{n} \text { and } t>0 .
$$

We establish a pointwise representation of the Laplace transform type multiplier $T_{m}^{\mathcal{L}}$ as a principal value integral operator and we prove $L^{p}$-boundedness properties of $T_{m}^{\mathcal{L}}$. As usual by $C_{c}^{\infty}\left(\mathbf{R}^{n}\right)$ we denote the space of smooth functions with compact support in $\mathbf{R}^{n}$.

Theorem 1. Suppose that $m(\lambda)=\lambda \int_{0}^{\infty} e^{-\lambda t} \phi(t) d t, \lambda \in(0, \infty)$, where $\phi \in$ $L^{\infty}(0, \infty)$. Then, for every $f \in C_{c}^{\infty}\left(\mathbf{R}^{n}\right)$,

$$
T_{m}^{\mathcal{L}}(f)(x)=\lim _{\varepsilon \rightarrow 0^{+}}\left(\alpha(\varepsilon) f(x)+\int_{|x-y|>\varepsilon} K_{\phi}^{\mathcal{L}}(x, y) f(y) d y\right), \quad \text { a.e. } x \in \mathbf{R}^{n},
$$

where

$$
K_{\phi}^{\mathcal{L}}(x, y)=-\int_{0}^{\infty} \phi(t) \frac{\partial}{\partial t} W_{t}^{\mathcal{L}}(x, y) d t, \quad x, y \in \mathbf{R}^{n}, x \neq y,
$$

and $\alpha$ is a certain measurable bounded function on $(0, \infty)$. Moreover, if there exists the limit $\lim _{t \rightarrow 0^{+}} \phi(t)=\phi\left(0^{+}\right)$, then

$$
T_{m}^{\mathcal{L}}(f)(x)=\phi\left(0^{+}\right) f(x)+\lim _{\varepsilon \rightarrow 0^{+}} \int_{|x-y|>\varepsilon} K_{\phi}^{\mathcal{L}}(x, y) f(y) d y, \quad \text { a.e. } x \in \mathbf{R}^{n} .
$$


Theorem 2. Suppose that $m(\lambda)=\lambda \int_{0}^{\infty} e^{-\lambda t} \phi(t) d t, \lambda \in(0, \infty)$, where $\phi \in$ $L^{\infty}(0, \infty)$. Then $T_{m}^{\mathcal{L}}$ can be extended to $L^{p}\left(\mathbf{R}^{n}\right)$ as a bounded operator from $L^{p}\left(\mathbf{R}^{n}\right)$ into itself, for every $1<p<\infty$, and as a bounded operator from $L^{1}\left(\mathbf{R}^{n}\right)$ into $L^{1, \infty}\left(\mathbf{R}^{n}\right)$. Moreover, this extension can be given by (1) and, when the limit $\lim _{t \rightarrow 0^{+}}$ $\phi(t)=\phi\left(0^{+}\right)$exists, by (2), for every $f \in L^{p}\left(\mathbf{R}^{n}\right), 1 \leq p<\infty$.

Note that since the semigroup of operators $\left\{W_{t}^{\mathcal{L}}\right\}_{t>0}$ is not conservative, the $L^{p}$-boundedness, $1<p<\infty$, of the Laplace transform type multipliers $T_{m}^{\mathcal{L}}$ can not be deduced from the result established in [17, p. 121]. The harmonic analysis operators (maximal operators, Riesz transforms and Littlewood-Paley g-functions) in the Schrödinger setting have been studied in $L^{p}$-spaces by several authors in last years (see for instance, [1], [8] and [16]). In order to show Theorems 1 and 2, inspired in the procedure developed by Shen [16] to analyze Riesz transforms, we take advantage that $\mathcal{L}$ is a "nice" perturbation of the Laplacian operator $-\Delta$. This fact allows us to write the multipliers in the Schrödinger setting, in some local sense, as perturbations of the corresponding multipliers associated to the Laplacian.

In the localization of our operators the function $\rho$ defined in $[16$, p. 516$]$ by

$$
\rho(x)=\sup \left\{r>0: \frac{1}{r^{n-2}} \int_{B(x, r)} V(y) d y \leq 1\right\}, \quad x \in \mathbf{R}^{n},
$$

plays an important role. The main properties of this function $\rho$ can be encountered in $[16, \S 1]$. We also use several properties of the heat kernel $W_{t}^{\mathcal{L}}(x, y)$ associated to the Schrödinger operator $\mathcal{L}$ that can be found, for instance, in [8].

A Banach space $B$ is said to be UMD when the Hilbert transform $H$ defined in a natural way on $L^{p}\left(\mathbf{R}^{n}\right) \otimes B$ can be extended to $L^{p}\left(\mathbf{R}^{n}, B\right)$ as a bounded operator from $L^{p}\left(\mathbf{R}^{n}, B\right)$ into itself for some (equivalently, for every) $1<p<\infty$ (see [4] and [5]). Here, for every $1 \leq p<\infty$, by $L^{p}\left(\mathbf{R}^{n}, B\right)$ we represent the Bochner-Lebesgue space of exponent $p$. UMD property is related to geometric properties of Banach spaces ([6]). In last years several authors have established connections between geometry of Banach spaces and harmonic analysis. In particular, characterizations of UMD, convexity or smoothness properties of a Banach space have been given in terms of $L^{p}$-boundedness of certain singular integrals or Littlewood-Paley g-functions ([1], [12], [13], [14] and [20]). Guerre-Delabrière ([11]) characterized the Banach spaces having the UMD property by the $L^{p}$-boundedness of the imaginary power $\left(-\frac{d^{2}}{d x^{2}}\right)^{i \gamma}$, $\gamma \in \mathbf{R}$, of the Laplacian in one dimension. Here, inspired in [11], we characterize the Banach spaces having the UMD property by the $L^{p}$-boundedness of the imaginary power $\mathcal{L}^{i \gamma}, \gamma \in \mathbf{R}$, of the Schrödinger operator.

Let $\gamma \in \mathbf{R}$. We denote by $m_{\gamma}$ the function $m_{\gamma}(\lambda)=\lambda^{i \gamma}, \lambda \in(0, \infty)$. It is clear that $m_{\gamma}(\lambda)=\lambda \int_{0}^{\infty} e^{-\lambda t} \phi_{\gamma}(t) d t, \lambda \in(0, \infty)$, where $\phi_{\gamma}(t)=t^{-i \gamma} / \Gamma(1-i \gamma), t \in(0, \infty)$. We define, as usual, the imaginary power $\mathcal{L}^{i \gamma}$ of $\mathcal{L}$ by

$$
\mathcal{L}^{i \gamma}=T_{m_{\gamma}}^{\mathcal{L}}
$$

For every $\varepsilon>0$ and every $f \in L^{p}\left(\mathbf{R}^{n}, B\right), 1 \leq p<\infty$, we consider the truncation

$$
\mathcal{L}_{\varepsilon}^{i \gamma}(f)(x)=\alpha(\varepsilon) f(x)+\int_{|x-y|>\varepsilon} K_{\phi_{\gamma}}^{\mathcal{L}}(x, y) f(y) d y
$$


where $\alpha$ and $K_{\phi_{\gamma}}^{\mathcal{L}}(x, y)$ are as in Theorem 1, and the maximal operator associated with $\mathcal{L}^{i \gamma}$ is defined by

$$
\mathcal{L}_{*}^{i \gamma}(f)=\sup _{\varepsilon>0}\left\|\mathcal{L}_{\varepsilon}^{i \gamma}(f)\right\|_{B}
$$

According to Theorem $2, \mathcal{L}^{i \gamma}$ can be extended to $L^{p}\left(\mathbf{R}^{n}\right)$ as a bounded operator from $L^{p}\left(\mathbf{R}^{n}\right)$ into itself, for every $1<p<\infty$. If $B$ is a Banach space we define $\mathcal{L}^{i \gamma}$ on $L^{p}\left(\mathbf{R}^{n}\right) \otimes B, 1<p<\infty$, in a natural way.

Theorem 3. Let $B$ be a Banach space. Then, the following properties are equivalent:

(i) $B$ is a UMD space.

(ii) For every $\gamma \in \mathbf{R}$ and for some (equivalently, for any) $1<p<\infty$, the operator $\mathcal{L}^{i \gamma}$ can be extended to $L^{p}\left(\mathbf{R}^{n}, B\right)$ as a bounded operator from $L^{p}\left(\mathbf{R}^{n}, B\right)$ into itself.

(iii) For every $\gamma \in \mathbf{R}$, the operator $\mathcal{L}^{i \gamma}$ can be extended to $L^{1}\left(\mathbf{R}^{n}, B\right)$ as a bounded operator from $L^{1}\left(\mathbf{R}^{n}, B\right)$ into $L^{1, \infty}\left(\mathbf{R}^{n}, B\right)$.

(iv) For every $\gamma \in \mathbf{R}$ and every $f \in L^{1}\left(\mathbf{R}^{n}, B\right), \mathcal{L}_{*}^{i \gamma}(f)(x)<\infty$, a.e. $x \in \mathbf{R}^{n}$.

(v) For every $\gamma \in \mathbf{R}$ and every $f \in L^{1}\left(\mathbf{R}^{n}, B\right)$, there exists the limit $\lim _{\varepsilon \rightarrow 0^{+}}$ $\mathcal{L}_{\varepsilon}^{i \gamma}(f)(x)$, a.e. $x \in \mathbf{R}^{n}$.

In order to prove Theorem 3 we need to establish previously the following extension of [11, Theorem, p. 402]. The maximal operator $(-\Delta)_{*}^{i \gamma}$ is defined on $L^{1}\left(\mathbf{R}^{n}, B\right)$ as follows

$$
(-\Delta)_{*}^{i \gamma}(f)=\sup _{\varepsilon>0}\left\|(-\Delta)_{\varepsilon}^{i \gamma}(f)\right\|_{B}, \quad f \in L^{1}\left(\mathbf{R}^{n}, B\right),
$$

and, for every $\varepsilon>0$, the truncation $(-\Delta)_{\varepsilon}^{i \gamma}$ is defined as in (3) by replacing $\mathcal{L}$ by the Laplacian operator $-\Delta$.

Theorem 4. Let $B$ be a Banach space. Then, the following assertions are equivalent:

(i) $B$ is a UMD space.

(ii) For every $\gamma \in \mathbf{R}$ and for some (equivalently, for any) $1<p<\infty$, the operator $(-\Delta)^{i \gamma}$ can be extended to $L^{p}\left(\mathbf{R}^{n}, B\right)$ as a bounded operator from $L^{p}\left(\mathbf{R}^{n}, B\right)$ into itself.

(iii) For every $\gamma \in \mathbf{R}$, the operator $(-\Delta)^{i \gamma}$ can be extended to $L^{1}\left(\mathbf{R}^{n}, B\right)$ as a bounded operator from $L^{1}\left(\mathbf{R}^{n}, B\right)$ into $L^{1, \infty}\left(\mathbf{R}^{n}, B\right)$.

(iv) For every $\gamma \in \mathbf{R}$ and every $f \in L^{1}\left(\mathbf{R}^{n}, B\right),(-\Delta)_{*}^{i \gamma}(f)(x)<\infty$, a.e. $x \in \mathbf{R}^{n}$.

(v) For every $\gamma \in \mathbf{R}$ and every $f \in L^{1}\left(\mathbf{R}^{n}, B\right)$, there exists the limit $\lim _{\varepsilon \rightarrow 0^{+}}$ $(-\Delta)_{\varepsilon}^{i \gamma}(f)(x)$, a.e. $x \in \mathbf{R}^{n}$.

It is convenient to note that the equivalence (i) $\Leftrightarrow$ (ii) in Theorem 4 extends [11, Theorem, p. 402] to higher dimensions, and the equivalences (i) $\Leftrightarrow$ (iii) $\Leftrightarrow$ (iv) $\Leftrightarrow$ (v) are, as far we know, news.

This paper is organized as follows. In Section 2 we present a proof of Theorem 1. Theorems 2, 3 and 4 are proved in Section 3. Finally, we present in the Appendix, for the sake of completeness, a proof of a version of Theorem 1 in the Laplacian (classical) case.

Throughout this paper by $C$ and $c$ we always denote positive constants that can change in each occurrence. 


\section{Proof of Theorem 1}

Assume that $\phi \in L^{\infty}(0, \infty)$ and define the function $m$ as follows

$$
m(\lambda)=\lambda \int_{0}^{\infty} e^{-\lambda v} \phi(v) d v, \quad \lambda \in(0, \infty) .
$$

As it was commented $m$ is continuous and bounded in $(0, \infty)$. The spectral multiplier $T_{m}^{\mathcal{L}}$ in the Schrödinger setting associated with $m$ is defined by

$$
T_{m}^{\mathcal{L}}(f)=\int_{(0, \infty)} m(\lambda) E_{\mathcal{L}}(d \lambda) f, \quad f \in L^{2}\left(\mathbf{R}^{n}\right)
$$

where $E_{\mathcal{L}}$ represents the spectral measure for the Schrödinger operator $\mathcal{L}$. It is well known that $T_{m}^{\mathcal{L}}$ is a bounded operator from $L^{2}\left(\mathbf{R}^{n}\right)$ into itself.

We are going to prove Theorem 1 . Let $f, g \in C_{c}^{\infty}\left(\mathbf{R}^{n}\right)$. We can write

$$
\left\langle T_{m}^{\mathcal{L}}(f), g\right\rangle=\left\langle\int_{(0, \infty)} m(\lambda) E_{\mathcal{L}}(d \lambda) f, g\right\rangle=\int_{(0, \infty)} m(\lambda) d \mu_{f, g ; \mathcal{L}}(\lambda),
$$

where by $\mu_{f, g ; \mathcal{L}}$ we denote the measure defined by

$$
\mu_{f, g ; \mathcal{L}}(A)=\left\langle E_{\mathcal{L}}(A) f, g\right\rangle,
$$

for every Borel set $A \subset(0, \infty)$. The set function $\mu_{f, g ; \mathcal{L}}$ is a complex measure on $(0, \infty)$ satisfying that $\left|\mu_{f, g ; \mathcal{L}}\right|((0, \infty)) \leq\|f\|_{2}\|g\|_{2}$, where $\left|\mu_{f, g ; \mathcal{L}}\right|$ represents the total variation measure of $\mu_{f, g ; \mathcal{L}}$.

We have that

$$
\begin{aligned}
\left\langle T_{m}^{\mathcal{L}}(f), g\right\rangle & =\int_{(0, \infty)} \lambda \int_{0}^{\infty} e^{-\lambda v} \phi(v) d v d \mu_{f, g ; \mathcal{L}}(\lambda)=\int_{0}^{\infty} \phi(v) \int_{(0, \infty)} \lambda e^{-\lambda v} d \mu_{f, g ; \mathcal{L}}(\lambda) d v \\
& =\int_{0}^{\infty} \phi(v) \int_{(0, \infty)}\left(-\frac{\partial}{\partial v}\right)\left(e^{-\lambda v}\right) d \mu_{f, g ; \mathcal{L}}(\lambda) d v .
\end{aligned}
$$

Here, we can interchange the order of integration because

$$
\int_{(0, \infty)} \int_{0}^{\infty} \lambda e^{-\lambda v}|\phi(v)| d v d\left|\mu_{f, g ; \mathcal{L}}\right|(\lambda) \leq\|\phi\|_{\infty}\left|\mu_{f, g ; \mathcal{L}}\right|((0, \infty))<\infty .
$$

Since

$$
\left|\frac{e^{-\lambda(v+h)}-e^{-\lambda v}}{h}\right| \leq \lambda e^{\lambda(|h|-v)} \leq \lambda e^{-\lambda v / 2}, \quad v, \lambda>0 \text { and }|h|<\frac{v}{2}
$$

and

$$
\int_{(0, \infty)} \lambda e^{-\lambda v / 2} d\left|\mu_{f, g ; \mathcal{L}}\right|(\lambda) \leq \frac{2}{v}\left|\mu_{f, g ; \mathcal{L}}\right|((0, \infty))<\infty, \quad v>0,
$$

we can differentiate under the integral sign and write

$$
\begin{aligned}
\left\langle T_{m}^{\mathcal{L}}(f), g\right\rangle & =\int_{0}^{\infty} \phi(v)\left(-\frac{d}{d v}\right) \int_{(0, \infty)} e^{-\lambda v} d \mu_{f, g ; \mathcal{L}}(\lambda) d v \\
& =\int_{0}^{\infty} \phi(v)\left(-\frac{d}{d v}\right)\left\langle W_{v}^{\mathcal{L}}(f), g\right\rangle d v \\
& =\int_{0}^{\infty} \phi(v)\left(-\frac{d}{d v}\right) \int_{\mathbf{R}^{n}} \int_{\mathbf{R}^{n}} W_{v}^{\mathcal{L}}(x, y) f(y) d y \overline{g(x)} d x d v .
\end{aligned}
$$


We have that

$$
\int_{\mathbf{R}^{n}} \int_{\mathbf{R}^{n}}\left|W_{v}^{\mathcal{L}}(x, y)-W_{v}(x, y)\right||f(y)||g(x)| d y d x<\infty, \quad v \in(0, \infty),
$$

and

$$
\int_{\mathbf{R}^{n}} \int_{\mathbf{R}^{n}}\left|\frac{\partial}{\partial v}\left(W_{v}^{\mathcal{L}}(x, y)-W_{v}(x, y)\right)\right||f(y)||g(x)| d y d x<\infty, \quad v \in(0, \infty) .
$$

Indeed, according to $[8,(2.2)$ and $(2.7)]$ it follows that

$$
\begin{aligned}
& \int_{\mathbf{R}^{n}} \int_{\mathbf{R}^{n}}\left|W_{v}^{\mathcal{L}}(x, y)-W_{v}(x, y)\right||f(y)||g(x)| d y d x \\
& \quad+\int_{\mathbf{R}^{n}} \int_{\mathbf{R}^{n}}\left|\frac{\partial}{\partial v}\left(W_{v}^{\mathcal{L}}(x, y)-W_{v}(x, y)\right)\right||f(y)||g(x)| d y d x \\
& \leq C \frac{1+v}{v^{n / 2+1}} \int_{\mathbf{R}^{n}} \int_{\mathbf{R}^{n}} e^{-c \frac{|x-y|^{2}}{v}}|f(y)||g(x)| d y d x \\
& \leq C \frac{1+v}{v^{n / 2+1}} \int_{\mathbf{R}^{n}}|f(y)| d y \int_{\mathbf{R}^{n}}|g(x)| d x<\infty, \quad v \in(0, \infty) .
\end{aligned}
$$

Hence, the function

$$
\psi(v)=\int_{\mathbf{R}^{n}} \int_{\mathbf{R}^{n}}\left(W_{v}^{\mathcal{L}}(x, y)-W_{v}(x, y)\right) f(y) \overline{g(x)} d y d x, \quad v \in(0, \infty),
$$

is differentiable in $(0, \infty)$ and

$$
\frac{d}{d v} \psi(v)=\int_{\mathbf{R}^{n}} \int_{\mathbf{R}^{n}} \frac{\partial}{\partial v}\left(W_{v}^{\mathcal{L}}(x, y)-W_{v}(x, y)\right) f(y) \overline{g(x)} d y d x, \quad v \in(0, \infty) .
$$

We can write

$$
\begin{aligned}
\left\langle T_{m}^{\mathcal{L}}(f), g\right\rangle= & \int_{0}^{\infty} \phi(v)\left(-\frac{d}{d v}\right) \int_{\mathbf{R}^{n}} \int_{\mathbf{R}^{n}} W_{v}(x, y) f(y) d y \overline{g(x)} d x d v \\
& +\int_{0}^{\infty} \phi(v) \int_{\mathbf{R}^{n}} \int_{\mathbf{R}^{n}}\left(-\frac{\partial}{\partial v}\right)\left(W_{v}^{\mathcal{L}}(x, y)-W_{v}(x, y)\right) f(y) \overline{g(x)} d y d x d v .
\end{aligned}
$$

Also, we have that

$$
\int_{\mathbf{R}^{n}} \int_{\mathbf{R}^{n}} \int_{0}^{\infty}|\phi(v)|\left|\frac{\partial}{\partial v}\left(W_{v}^{\mathcal{L}}(x, y)-W_{v}(x, y)\right)\right||f(y)||\overline{g(x)}| d v d x d y<\infty
$$

and

$$
\int_{\mathbf{R}^{n}} \int_{0}^{\infty}\left|\frac{\partial}{\partial v}\left(W_{v}^{\mathcal{L}}(x, y)-W_{v}(x, y)\right)\right||f(y)||\phi(v)| d v d y<\infty .
$$

Indeed, to see (6) we write

$$
\begin{aligned}
& \int_{\mathbf{R}^{n}} \int_{0}^{\infty}\left|\frac{\partial}{\partial v}\left(W_{v}^{\mathcal{L}}(x, y)-W_{v}(x, y)\right)\right||f(y)||\phi(v)| d v d y \\
& \leq\|\phi\|_{\infty}\left(\int_{\mathbf{R}^{n}} \int_{0}^{\rho(x)^{2}}+\int_{\mathbf{R}^{n}} \int_{\rho(x)^{2}}^{\infty}\right)\left|\frac{\partial}{\partial v}\left(W_{v}^{\mathcal{L}}(x, y)-W_{v}(x, y)\right)\right||f(y)| d v d y \\
& =B_{1}(x)+B_{2}(x) .
\end{aligned}
$$


According to $[8,(2.7)]$ we get

$$
B_{2}(x) \leq C \int_{\mathbf{R}^{n}} \int_{\rho(x)^{2}}^{\infty} \frac{e^{-c \frac{|x-y|^{2}}{v}}}{v^{\frac{n}{2}+1}}|f(y)| d v d y \leq \frac{C}{\rho(x)^{n}}, \quad x \in \mathbf{R}^{n} .
$$

Since $0<\rho(x)<\infty$, it follows that $B_{2}(x)<\infty, x \in \mathbf{R}^{n}$.

By proceeding as in [3, p. 15-17] we can obtain that

$$
B_{1}(x) \leq C\|f\|_{\infty}, \quad x \in \mathbf{R}^{n} .
$$

Thus, we have proved (6). Moreover, by using [16, Lemma 1.4], (7) and (8) imply also (5). Then (4) can be written

$$
\begin{aligned}
\left\langle T_{m}^{\mathcal{L}}(f), g\right\rangle= & \int_{0}^{\infty} \phi(v)\left(-\frac{d}{d v}\right) \int_{\mathbf{R}^{n}} \int_{\mathbf{R}^{n}} W_{v}(x, y) f(y) d y \overline{g(x)} d x d v \\
& +\int_{\mathbf{R}^{n}}\left(\lim _{\varepsilon \rightarrow 0^{+}} \int_{|x-y|>\varepsilon}\left(K_{\phi}^{\mathcal{L}}(x, y)-K_{\phi}(x, y)\right) f(y) d y\right) \overline{g(x)} d x
\end{aligned}
$$

where

$$
K_{\phi}^{\mathcal{L}}(x, y)=-\int_{0}^{\infty} \phi(v) \frac{\partial}{\partial v} W_{v}^{\mathcal{L}}(x, y) d v, \quad x, y \in \mathbf{R}^{n}, x \neq y
$$

and

$$
K_{\phi}(x, y)=-\int_{0}^{\infty} \phi(v) \frac{\partial}{\partial v} W_{v}(x, y) d v, \quad x, y \in \mathbf{R}^{n}, x \neq y .
$$

On the other hand, as above we can see that

$$
\left\langle T_{m}(f), g\right\rangle=\int_{0}^{\infty} \phi(v)\left(-\frac{d}{d v}\right) \int_{\mathbf{R}^{n}} \int_{\mathbf{R}^{n}} W_{v}(x, y) f(y) d y \overline{g(x)} d x d v .
$$

where $T_{m}$ represents the spectral multiplier associated with $-\Delta$ defined by $m$. Moreover, we can write

$$
T_{m}(f)(x)=\lim _{\varepsilon \rightarrow 0^{+}}\left(\alpha(\varepsilon) f(x)+\int_{|x-y|>\varepsilon} K_{\phi}(x, y) f(y) d y\right), \quad \text { a.e. } x \in \mathbf{R}^{n} .
$$

where

$$
\alpha(\varepsilon)=\frac{1}{\Gamma\left(\frac{n}{2}\right)} \int_{0}^{\infty} \phi\left(\frac{\varepsilon^{2}}{4 u}\right) e^{-u} u^{\frac{n}{2}-1} d u, \quad \varepsilon>0 .
$$

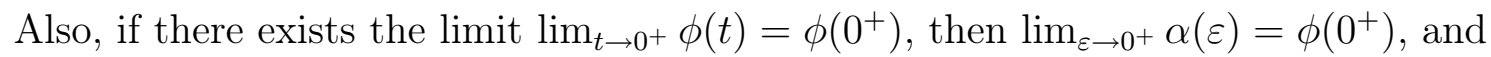

$$
T_{m}(f)(x)=\phi\left(0^{+}\right) f(x)+\lim _{\varepsilon \rightarrow 0^{+}} \int_{|x-y|>\varepsilon} K_{\phi}(x, y) f(y) d y, \quad \text { a.e. } x \in \mathbf{R}^{n} .
$$

Although we are sure that the properties (11) and (13) are known, we include in the appendix complete proofs for these properties of $T_{m}$, for the sake the interested reader.

By combining (9), (10), (11) and (13) we obtain that

$$
T_{m}^{\mathcal{L}}(f)(x)=\lim _{\varepsilon \rightarrow 0^{+}}\left(\alpha(\varepsilon) f(x)+\int_{|x-y|>\varepsilon} K_{\phi}^{\mathcal{L}}(x, y) f(y) d y\right), \quad \text { a.e. } x \in \mathbf{R}^{n},
$$

and

$$
T_{m}^{\mathcal{L}}(f)(x)=\phi\left(0^{+}\right) f(x)+\lim _{\varepsilon \rightarrow 0^{+}} \int_{|x-y|>\varepsilon} K_{\phi}^{\mathcal{L}}(x, y) f(y) d y, \quad \text { a.e. } x \in \mathbf{R}^{n}
$$


provided that there exists the limit $\phi\left(0^{+}\right)=\lim _{t \rightarrow 0^{+}} \phi(t)$.

\section{Proof of Theorems 2, 3 and 4}

Guerre-Delabrière [11, Theorem, p. 402] established that a Banach space $B$ is UMD if, only if, for every $\gamma \in \mathbf{R}$, the operator $\left(-\frac{d^{2}}{d x^{2}}\right)^{i \gamma}$ can be extended to $L^{p}(\mathbf{R}, B)$ into itself, for some (equivalently, for any) $1<p<\infty$. In the proof of [11, Theorem, p. 402] a vector valued version of a classical transference result was used. form

Assume that $B$ is a Banach space and $\gamma \in \mathbf{R}$. The operator $\left(-\frac{d^{2}}{d x^{2}}\right)^{i \gamma}$ takes the

$$
\left(-\frac{d^{2}}{d x^{2}}\right)^{i \gamma} f=\left(|y|^{2 i \gamma} \hat{f}\right)^{\swarrow}, \quad f \in L^{2}(\mathbf{R}),
$$

where $\hat{f}$ denotes the Fourier transform of $f$ and $\check{f}$ the inverse Fourier transform of $f$. If $f \in L^{1}(\mathbf{R})$ we define

$$
\hat{f}(y)=\int_{\mathbf{R}} e^{-i x y} f(x) d x, \quad y \in \mathbf{R},
$$

and

$$
\check{f}(y)=\frac{1}{2 \pi} \int_{\mathbf{R}} e^{i x y} f(x) d x, \quad y \in \mathbf{R} .
$$

As it is well known the Fourier transform can be extended from $L^{1}(\mathbf{R}) \cap L^{2}(\mathbf{R})$ to $L^{2}(\mathbf{R})$ as a bijective bounded operator from $L^{2}(\mathbf{R})$ into itself. The operator $\left(-\frac{d^{2}}{d x^{2}}\right)^{i \gamma}$ is bounded from $L^{p}(\mathbf{R})$ into itself, for every $1<p<\infty$. If $1<p<\infty$ and $f \in L^{p}(\mathbf{R}) \otimes B$, that is, $f=\sum_{j=1}^{r} \beta_{j} f_{j}$, where $\beta_{j} \in B, f_{j} \in L^{p}(\mathbf{R}), j=1, \ldots, r \in \mathbf{N}$, we define, as usual,

$$
\left(-\frac{d^{2}}{d x^{2}}\right)^{i \gamma}(f)=\sum_{j=1}^{r} \beta_{j}\left(-\frac{d^{2}}{d x^{2}}\right)^{i \gamma}\left(f_{j}\right)
$$

We also consider the operator $\left(-\frac{d^{2}}{d x^{2}}\right)_{\mid \mathbf{T}}^{i \gamma}$, where $\mathbf{T}=[0,2 \pi)$ denotes the onedimensional torus, defined by $\left(-\frac{d^{2}}{d x^{2}}\right)_{\mid \mathbf{T}}^{i \gamma}(g)(x)=\sum_{j \in \mathbf{Z}, j \neq 0}|j|^{2 i \gamma} c_{j}(g) e^{i j x}, x \in(0,2 \pi) \quad$ and $\quad g \in L^{p}(\mathbf{T}), 1<p<\infty$, being $c_{j}(g)=\frac{1}{2 \pi} \int_{0}^{2 \pi} g(\theta) e^{-i j \theta} d \theta, j \in \mathbf{Z}$. The operator $\left(-\frac{d^{2}}{d x^{2}}\right)_{\mid \mathbf{T}}^{i \gamma}$ is bounded from $L^{p}(\mathbf{T})$ into itself, $1<p<\infty$. If $1<p<\infty$ and $g \in L^{p}(\mathbf{T}) \otimes B$, that is, $g=\sum_{j=1}^{r} \beta_{j} g_{j}$, where $\beta_{j} \in B, g_{j} \in L^{p}(\mathbf{T}), j=1, \ldots, r \in \mathbf{N}$, we define

$$
\left(-\frac{d^{2}}{d x^{2}}\right)_{\mid \mathbf{T}}^{i \gamma}(g)=\sum_{j=1}^{r} \beta_{j}\left(-\frac{d^{2}}{d x^{2}}\right)_{\mid \mathbf{T}}^{i \gamma}\left(g_{j}\right) \text {. }
$$

Guerre-Delabrière ([11, p. 402]) showed that if $\left(-\frac{d^{2}}{d x^{2}}\right)_{\mid \mathbf{T}}^{i \gamma}$ can be extended to $L^{2}(\mathbf{T}, B)$ as a bounded operator from $L^{2}(\mathbf{T}, B)$ into itself, then $B$ is UMD. Moreover, she used a vector valued transference result (see [7] for the scalar result) that implies 
that $\left(-\frac{d^{2}}{d x^{2}}\right)_{\mid \mathbf{T}}^{i \gamma}$ can be extended to $L^{2}(\mathbf{T}, B)$ as a bounded operator from $L^{2}(\mathbf{T}, B)$ into itself, provided that $\left(-\frac{d^{2}}{d x^{2}}\right)^{i \gamma}$ can be extended to $L^{2}(\mathbf{R}, B)$ as a bounded operator from $L^{2}(\mathbf{R}, B)$ into itself. Note that, by using vector valued CalderónZygmund theory $([15])$ we can see that $\left(-\frac{d^{2}}{d x^{2}}\right)^{i \gamma}$ can be extended to $L^{p}(\mathbf{R}, B)$ as a bounded operator from $L^{p}(\mathbf{R}, B)$ into itself, for some $1<p<\infty$, if and only if $\left(-\frac{d^{2}}{d x^{2}}\right)^{i \gamma}$ can be extended to $L^{2}(\mathbf{R}, B)$ as a bounded operator from $L^{2}(\mathbf{R}, B)$ into itself.

The operators $(-\Delta)^{i \gamma}$ (respectively, $\left.(-\Delta)_{\mid \mathbf{T}^{n}}^{i \gamma}\right)$ are defined on $L^{p}\left(\mathbf{R}^{n}\right)$ and $L^{p}\left(\mathbf{R}^{n}\right)$ $\otimes B$ (respectively, on $L^{p}\left(\mathbf{T}^{n}\right)$ and $\left.L^{p}\left(\mathbf{T}^{n}\right) \otimes B\right), 1<p<\infty$, in the natural way.

3.1. Proof of Theorem 4. (i) $\Rightarrow$ (ii). It is a consequence of [21, Proposition 3].

(ii) $\Rightarrow$ (i). We show this part by adapting standard transference arguments to a vector valued setting. For the sake of completeness we include the proof.

Let $\gamma \in \mathbf{R}$ and $1<p<\infty$. Suppose that the operator $(-\Delta)^{i \gamma}$ can be extended to $L^{p}\left(\mathbf{R}^{n}, B\right)$ as a bounded operator from $L^{p}\left(\mathbf{R}^{n}, B\right)$ into itself. We choose an even smooth function on $\mathbf{R}$ such that $\phi(x)=1,|x| \leq 1 / 4$, and $\phi(x)=0,|x| \geq 1 / 2$. We split the operator $(-\Delta)^{i \gamma}$ as follows

$$
\begin{aligned}
(-\Delta)^{i \gamma}(f) & =\left(\phi\left(|x|^{2}\right)|x|^{2 i \gamma} \hat{f}\right)^{\swarrow}+\left(\left(1-\phi\left(|x|^{2}\right)\right)|x|^{2 i \gamma} \hat{f}\right)^{\swarrow} \\
& =A_{1}(f)+A_{2}(f), \quad f \in C_{c}^{\infty}\left(\mathbf{R}^{n}\right) \otimes B .
\end{aligned}
$$

Here, $\hat{h}$ denotes the Fourier transform of $h$ and $\breve{h}$ the inverse Fourier transform of $h$ in $\mathbf{R}^{n}$, defined, for every $h \in L^{1}\left(\mathbf{R}^{n}\right)$, by

$$
\hat{h}(y)=\int_{\mathbf{R}^{n}} e^{-i x y} h(x) d x, \quad y \in \mathbf{R}^{n},
$$

and

$$
\check{h}(y)=\frac{1}{(2 \pi)^{n}} \int_{\mathbf{R}^{n}} e^{i x y} h(x) d x, \quad y \in \mathbf{R}^{n} .
$$

Also, we consider the function $\varphi(x)=\phi\left(|x|^{2}\right), x \in \mathbf{R}^{n}$, and the Fourier multiplier $T_{\varphi}$ defined by

$$
T_{\varphi}(f)=(\varphi \hat{f})^{\swarrow}, \quad f \in C_{c}^{\infty}\left(\mathbf{R}^{n}\right) \otimes B,
$$

in a natural way. Since $\hat{\varphi} \in L^{1}\left(\mathbf{R}^{n}\right), T_{\varphi}$ can be extended to $L^{p}\left(\mathbf{R}^{n}, B\right)$ as a bounded operator from $L^{p}\left(\mathbf{R}^{n}, B\right)$ into itself. Then, $A_{1}$ and therefore $A_{2}$ can be extended to $L^{p}\left(\mathbf{R}^{n}, B\right)$ as a bounded operator from $L^{p}\left(\mathbf{R}^{n}, B\right)$ into itself.

We denote by $\mathcal{P}\left(\mathbf{T}^{n}, X\right)$ the space of trigonometric polynomials of period $2 \pi$ on $\mathbf{T}^{n}$ with coefficients in a Banach space $X$. Let $P \in \mathcal{P}\left(\mathbf{T}^{n}, B\right)$ and $Q \in \mathcal{P}\left(\mathbf{T}^{n}, B^{\prime}\right)$, where $B^{\prime}$ is the dual space of $B$. Since $A_{2}$ can be extended to $L^{p}\left(\mathbf{R}^{n}, B\right)$ as a bounded operator from $L^{p}\left(\mathbf{R}^{n}, B\right)$ into itself, by proceeding as in the proof of [18, Theorem 3.8, p. 260] we have that

$$
\left|\int_{\mathbf{T}^{n}}\left\langle(-\Delta)_{\mid \mathbf{T}^{n}}^{i \gamma}(P)(x), Q(x)\right\rangle d x\right| \leq C\|P\|_{L^{p}\left(\mathbf{T}^{n}, B\right)}\|Q\|_{L^{p^{\prime}\left(\mathbf{T}^{n}, B^{\prime}\right)}},
$$

where $p^{\prime}$ is the exponent conjugated to $p$. 
By using [10, Lemma 2.3] we get

$$
\left\|(-\Delta)_{\mid \mathbf{T}^{n}}^{i \gamma}(P)\right\|_{L^{p}\left(\mathbf{T}^{n}, B\right)} \leq C\|P\|_{L^{p}\left(\mathbf{T}^{n}, B\right)} .
$$

Hence, $(-\Delta)_{\mid \mathbf{T}^{n}}^{i \gamma}$ can be extended to $L^{p}\left(\mathbf{T}^{n}, B\right)$ as a bounded operator from $L^{p}\left(\mathbf{T}^{n}, B\right)$ into itself.

In order to see that the operator $\left(-\frac{d^{2}}{d x^{2}}\right)_{\mid \mathbf{T}}^{i \gamma}$ can be extended to $L^{p}(\mathbf{T}, B)$ as a bounded operator from $L^{p}(\mathbf{T}, B)$ into itself, it is sufficient to use that $(-\Delta)_{\mid \mathbf{T}^{n}}^{i \gamma}$ can be extended to $L^{p}\left(\mathbf{T}^{n}, B\right)$ as a bounded operator from $L^{p}\left(\mathbf{T}^{n}, B\right)$ into itself, and to extend every function $f \in L^{p}(\mathbf{T})$ to $\mathbf{T}^{n}$ in the natural way, that is, defining $\tilde{f}\left(x_{1}, \ldots, x_{n}\right)=f\left(x_{1}\right),\left(x_{1}, \ldots, x_{n}\right) \in \mathbf{T}^{n}$.

According to [11, Theorem, p. 402] the above arguments allow us to conclude that (ii) $\Rightarrow$ (i).

(ii) $\Leftrightarrow$ (iii) $\Rightarrow$ (iv). These properties are true because, for every $\gamma \in \mathbf{R}$, the operator $(-\Delta)^{i \gamma}$ is a Calderón-Zygmund operator (see [15]).

(iv) $\Rightarrow$ (iii) and (iv) $\Rightarrow(\mathrm{v})$. Assume that (iv) holds. Let $\gamma \in \mathbf{R}$. For every $0<\varepsilon<1$, we define the operator $(-\Delta)_{(\varepsilon, 1 / \varepsilon)}^{i \gamma}$ on $L^{1}\left(\mathbf{R}^{n}, B\right)$ by

$$
(-\Delta)_{(\varepsilon, 1 / \varepsilon)}^{i \gamma}(f)(x)=\alpha(\varepsilon) f(x)+\int_{\varepsilon<|x-y|<1 / \varepsilon} K_{\phi_{\gamma}}(x, y) f(y) d y, \quad f \in L^{1}\left(\mathbf{R}^{n}, B\right),
$$

that is bounded from $L^{1}\left(\mathbf{R}^{n}, B\right)$ into itself. By using (iv) and continuity Banach principle $([9$, Proposition 1.4 , p. 529$])$ the maximal operator $(-\Delta)_{* *}^{i \gamma}$ defined by

$$
(-\Delta)_{* *}^{i \gamma}(f)=\sup _{\varepsilon \in(0,1)}\left\|(-\Delta)_{(\varepsilon, 1 / \varepsilon)}^{i \gamma}(f)\right\|_{B}, \quad f \in L^{1}\left(\mathbf{R}^{n}, B\right),
$$

is continuous from $L^{1}\left(\mathbf{R}^{n}, B\right)$ into $L^{0}\left(\mathbf{R}^{n}\right)$, where the space $L^{0}\left(\mathbf{R}^{n}\right)$ of all measurable functions is endowed with the local convergence in measure ([9, p. 528]). Since the function $\alpha$ is given by (12), the maximal operator $(-\Delta)_{*}^{i \gamma}$ is dilation and translation invariant. Moreover, by taking into account that $\left|K_{\phi_{\gamma}}(x, y)\right| \leq C|x-y|^{-n}, x, y \in \mathbf{R}^{n}$, $x \neq y,(-\Delta)_{*}^{i \gamma}$ is bounded from $L^{1}\left(\mathbf{R}^{n}, B\right)$ into $L^{0}\left(\mathbf{R}^{n}\right)$. Then, since every Banach space is of Rademacher type 1 , according to [14, Lemma 7.3], we deduce that $(-\Delta)_{*}^{i \gamma}$ is bounded from $L^{1}\left(\mathbf{R}^{n}, B\right)$ into $L^{1, \infty}\left(\mathbf{R}^{n}\right)$.

For every $f \in C_{c}^{\infty}\left(\mathbf{R}^{n}\right) \otimes B$, there exists the $\operatorname{limit}_{\lim _{\varepsilon \rightarrow 0^{+}}}(-\Delta)_{\varepsilon}^{i \gamma}(f)(x)$, a.e. $x \in \mathbf{R}^{n}$. Since $C_{c}^{\infty}\left(\mathbf{R}^{n}\right) \otimes B$ is a dense subspace of $L^{1}\left(\mathbf{R}^{n}, B\right)$, for every $f \in L^{1}\left(\mathbf{R}^{n}, B\right)$, there exists the limit $\lim _{\varepsilon \rightarrow 0^{+}}(-\Delta)_{\varepsilon}^{i \gamma}(f)(x)$, a.e. $x \in \mathbf{R}^{n}$, and $(-\Delta)^{i \gamma}$ can be extended to $L^{1}\left(\mathbf{R}^{n}, B\right)$ as a bounded operator from $L^{1}\left(\mathbf{R}^{n}, B\right)$ into $L^{1, \infty}\left(\mathbf{R}^{n}, B\right)$.

(v) $\Rightarrow$ (iv). It is clear.

3.2. Proof of Theorem 3. (i) $\Rightarrow$ (ii) and (i) $\Rightarrow$ (iii). Let $\gamma \in \mathbf{R}$. The imaginary power $\mathcal{L}^{i \gamma}$ of $\mathcal{L}$ (respectively, $(-\Delta)^{i \gamma}$ of $-\Delta$ ) is the spectral multiplier associated with $\mathcal{L}$ (respectively, $-\Delta$ ) defined by the function $m_{\gamma}(\lambda)=\lambda^{i \gamma}, \lambda \in(0, \infty)$. Note that $m_{\gamma}(\lambda)=\lambda \int_{0}^{\infty} e^{-\lambda t} \phi_{\gamma}(t) d t, \lambda \in(0, \infty)$, where $\phi_{\gamma}(t)=\frac{t^{-i \gamma}}{\Gamma(1-i \gamma)}, t \in(0, \infty)$.

Assume that $B$ is a Banach space and that $f=\sum_{j=1}^{d} \beta_{j} f_{j}$, where $f_{j} \in C_{c}^{\infty}\left(\mathbf{R}^{n}\right)$ and $\beta_{j} \in B, j=1, \ldots, d$. By Theorem 1 and (11), we have that

$$
T_{m_{\gamma}}^{\mathcal{L}}(f)(x)=\sum_{j=1}^{d} \beta_{j} \lim _{\varepsilon \rightarrow 0^{+}}\left(\alpha(\varepsilon) f_{j}(x)+\int_{|x-y|>\varepsilon} K_{\phi_{\gamma}}^{\mathcal{L}}(x, y) f_{j}(y) d y\right), \quad \text { a.e. } x \in \mathbf{R}^{n},
$$


and

$$
T_{m_{\gamma}}(f)(x)=\sum_{j=1}^{d} \beta_{j} \lim _{\varepsilon \rightarrow 0^{+}}\left(\alpha(\varepsilon) f_{j}(x)+\int_{|x-y|>\varepsilon} K_{\phi_{\gamma}}(x, y) f_{j}(y) d y\right), \quad \text { a.e. } x \in \mathbf{R}^{n} .
$$

We split the operator $T_{m_{\gamma}}$ as follow

$$
T_{m_{\gamma}}=T_{m_{\gamma}, g}+T_{m_{\gamma}, \ell},
$$

where $T_{m_{\gamma}, g}(f)(x)=\int_{|x-y| \geq \rho(x)} K_{\phi_{\gamma}}(x, y) f(y) d y, x \in \mathbf{R}^{n}$.

The operator $S_{m_{\gamma}}=T_{m_{\gamma}}^{\mathcal{L}}-T_{m_{\gamma}, \ell}$ can be extended to $L^{p}\left(\mathbf{R}^{n}, B\right)$ as a bounded operator from $L^{p}\left(\mathbf{R}^{n}, B\right)$ into itself, for every $1<p<\infty$. Indeed, we can write

$$
\begin{aligned}
S_{m_{\gamma}}(f)(x)= & \lim _{\varepsilon \rightarrow 0^{+}} \int_{\varepsilon<|x-y|<\rho(x)} f(y)\left(K_{\phi_{\gamma}}^{\mathcal{L}}(x, y)-K_{\phi_{\gamma}}(x, y)\right) d y \\
& -\int_{|x-y| \geq \rho(x)} f(y) \int_{0}^{\infty} \phi_{\gamma}(t) \frac{\partial}{\partial t} W_{t}^{\mathcal{L}}(x, y) d t d y \\
= & S_{m_{\gamma}, 1}(f)(x)+S_{m_{\gamma}, 2}(f)(x) .
\end{aligned}
$$

By proceeding as in [3, p. 15-17] we can get

$$
\left\|S_{m_{\gamma}, 1}(f)(x)\right\|_{B} \leq \int_{|x-y|<\rho(x)}\|f(y)\|_{B}\left|K_{\phi_{\gamma}}^{\mathcal{L}}(x, y)-K_{\phi_{\gamma}}(x, y)\right| d y \leq C \mathcal{M}(\|f\|)(x),
$$

$x \in \mathcal{R}^{n}$, and

$$
\begin{aligned}
\left\|S_{m_{\gamma}, 2} f(x)\right\|_{B} \leq & \int_{|x-y| \geq \rho(x)}\|f(y)\|_{B} \int_{0}^{\rho(x)^{2}}\left|\phi_{\gamma}(t)\right|\left|\frac{\partial}{\partial t} W_{t}^{\mathcal{L}}(x, y)\right| d t d y \\
& +\int_{|x-y| \geq \rho(x)}\|f(y)\|_{B} \int_{\rho(x)^{2}}^{\infty}\left|\phi_{\gamma}(t)\right|\left|\frac{\partial}{\partial t} W_{t}^{\mathcal{L}}(x, y)\right| d t d y \\
\leq & C \mathcal{M}\left(\|f\|_{B}\right)(x), \quad x \in \mathbf{R}^{n},
\end{aligned}
$$

because $\left\|\phi_{\gamma}\right\|_{\infty}=1 /|\Gamma(1-i \gamma)|$. Here and in the sequel $\mathcal{M}$ denotes the HardyLittlewood maximal function. Hence, by using the well known Maximal Theorem we conclude that the operator $S_{m_{\gamma}}$ can be extended to $L^{p}\left(\mathbf{R}^{n}, B\right)$ as a bounded operator from $L^{p}\left(\mathbf{R}^{n}, B\right)$ into itself, for every $1<p<\infty$.

Suppose now that $B$ is a $U M D$ Banach space. According to Theorem 4 the operator $T_{m_{\gamma}}$ can be extended to $L^{p}\left(\mathbf{R}^{n}, B\right)$ as a bounded operator from $L^{p}\left(\mathbf{R}^{n}, B\right)$ into itself, for every $1<p<\infty$, and to $L^{1}\left(\mathbf{R}^{n}, B\right)$ as a bounded operator from $L^{1}\left(\mathbf{R}^{n}, B\right)$ into $L^{1, \infty}\left(\mathbf{R}^{n}, B\right)$. For every $f \in L_{c}^{\infty}\left(\mathbf{R}^{n}\right) \otimes B$ we have that

$$
T_{m_{\gamma}}(f)(x)=\int_{\mathbf{R}^{n}} K_{\phi_{\gamma}}(x, y) f(y) d y, \quad \text { a.e. } x \notin \operatorname{supp} f .
$$

Moreover, $K_{\phi_{\gamma}}$ is a standard Calderón-Zygmund kernel, that is, there exists $C>0$ such that

and

$$
\left|K_{\phi_{\gamma}}(x, y)\right| \leq \frac{C}{|x-y|^{n}}, \quad x \neq y,
$$

$$
\sum_{j=1}^{n}\left(\left|\frac{\partial K_{\phi_{\gamma}}(x, y)}{\partial x_{j}}\right|+\left|\frac{\partial K_{\phi_{\gamma}}(x, y)}{\partial y_{j}}\right|\right) \leq \frac{C}{|x-y|^{n+1}}, \quad x \neq y .
$$


Then, by proceeding as in the scalar case (see [17, p. 34]), we can show that the maximal operator

$$
T_{m_{\gamma}}^{*}(f)(x)=\sup _{\varepsilon>0}\left\|\int_{|x-y|>\varepsilon} K_{\phi_{\gamma}}(x, y) f(y) d y\right\|_{B},
$$

is bounded from $L^{p}\left(\mathbf{R}^{n}, B\right)$ into $L^{p}\left(\mathbf{R}^{n}\right)$, for every $1<p<\infty$, and from $L^{1}\left(\mathbf{R}^{n}, B\right)$ into $L^{1, \infty}\left(\mathbf{R}^{n}\right)$.

It is clear that $\left\|T_{m_{\gamma}, g}(f)(x)\right\|_{B} \leq T_{m_{\gamma}}^{*}(f)(x), x \in \mathbf{R}^{n}$. Then, $T_{m_{\gamma}, g}$ is bounded from $L^{p}\left(\mathbf{R}^{n}, B\right)$ into itself, for every $1<p<\infty$, and from $L^{1}\left(\mathbf{R}^{n}, B\right)$ into $L^{1, \infty}\left(\mathbf{R}^{n}, B\right)$. Hence, since $T_{m_{\gamma}, \ell}=T_{m_{\gamma}}-T_{m_{\gamma}, g}$, we conclude that, $T_{m_{\gamma}, \ell}$, and then also $T_{m_{\gamma}}^{\mathcal{L}}$, are bounded from $L^{p}\left(\mathbf{R}^{n}, B\right)$ into itself, for every $1<p<\infty$, and from $L^{1}\left(\mathbf{R}^{n}, B\right)$ into $L^{1, \infty}\left(\mathbf{R}^{n}, B\right)$.

(ii) $\Rightarrow$ (i) and (iii) $\Rightarrow$ (i). Assume firstly that for a certain $1<p<\infty$ and every $\gamma \in \mathbf{R}$ the operator $T_{m_{\gamma}}^{\mathcal{L}}$ can be extended to $L^{p}\left(\mathbf{R}^{n}, B\right)$ as a bounded operator from $L^{p}\left(\mathbf{R}^{n}, B\right)$ into itself. Then, for every $\gamma \in \mathbf{R}$ the operator $T_{m_{\gamma}, \ell}$ can be extended to $L^{p}\left(\mathbf{R}^{n}, B\right)$ as a bounded operator from $L^{p}\left(\mathbf{R}^{n}, B\right)$ into itself. According to Theorem 4 in order to show that $B$ is a $U M D$ Banach space it is sufficient to see that for every $\gamma \in \mathbf{R}, T_{m_{\gamma}}$ can be extended to $L^{p}\left(\mathbf{R}^{n}, B\right)$ as a bounded operator from $L^{p}\left(\mathbf{R}^{n}, B\right)$ into itself.

Let $\gamma \in \mathbf{R}$. Suppose that $f \in C_{c}^{\infty}\left(\mathbf{R}^{n}\right)$ and $\operatorname{supp} f \subset B(0, M)$ for a certain $M>0$. For every $R>0$ we define $f_{R}(x)=f(\sqrt{R} x), x \in \mathbf{R}^{n}$. It is clear that, $\operatorname{supp} f_{R} \subset B\left(0, \frac{M}{\sqrt{R}}\right), R>0$.

In the following our arguments are inspired in the ones developed by AbuFalahah, Stinga and Torrea in [1]. We are going to show that for every $\lambda>0$ there exists $R>0$ such that $\operatorname{supp} f_{R} \subset B\left(\frac{x}{R}, \rho\left(\frac{x}{R}\right)\right)$, provided that $|x|<\lambda$. According to [16, Lemma 1.1] there exists $C_{1}>0$ for which

$$
\frac{1}{C_{1}} \rho(y) \leq \rho(x) \leq C_{1} \rho(y), \quad|x-y| \leq \rho(x) .
$$

Let $\lambda>0$. From [1, Lemma 3.5] we can find $R_{\lambda}>0$ such that $\left|y-\frac{x}{R}\right|<\rho\left(\frac{x}{R}\right)$, when $|y|<\frac{C_{1}^{2} \rho(0)}{2},|x|<\lambda$ and $R \geq R_{\lambda}$. We can take $R \geq \max \left\{R_{\lambda},\left(\frac{2 M}{\rho(0) C_{1}^{2}}\right)^{2}\right\}$. Then $\operatorname{supp} f_{R} \subseteq B\left(\frac{x}{R}, \rho\left(\frac{x}{R}\right)\right),|x|<\lambda$.

We can write, for every $R>0$,

$$
\begin{aligned}
& T_{m_{\gamma}}\left(f_{R}\right)\left(\frac{x}{\sqrt{R}}\right) \\
& =\lim _{\varepsilon \rightarrow 0^{+}}\left(\alpha(\varepsilon) f_{R}\left(\frac{x}{\sqrt{R}}\right)+\int_{\left|\frac{x}{\sqrt{R}}-y\right|>\varepsilon} f_{R}(y) \int_{0}^{\infty} \phi_{\gamma}(t)\left(-\frac{\partial}{\partial t}\right) W_{t}\left(\frac{x}{\sqrt{R}}, y\right) d t d y\right) \\
& =\lim _{\varepsilon \rightarrow 0^{+}}\left(\alpha(\varepsilon) f(x)+\int_{|x-u|>\varepsilon \sqrt{R}} f(u) \int_{0}^{\infty} \phi_{\gamma}(t)\left(-\frac{\partial}{\partial t}\right) W_{t}\left(\frac{x}{\sqrt{R}}, \frac{u}{\sqrt{R}}\right) d t \frac{d u}{R^{n / 2}}\right) \\
& =\lim _{\varepsilon \rightarrow 0^{+}}\left(\alpha(\varepsilon) f(x)+\left.R \int_{|x-u|>\varepsilon \sqrt{R}} f(u) \int_{0}^{\infty} \phi_{\gamma}(t)\left(-\frac{\partial}{\partial s} W_{s}(x, u)\right)\right|_{s=R t} d t d u\right) \\
& =\lim _{\varepsilon \rightarrow 0^{+}}\left(\alpha(\varepsilon) f(x)+\int_{|x-u|>\varepsilon \sqrt{R}} f(u) \int_{0}^{\infty} \phi_{\gamma}\left(\frac{s}{R}\right)\left(-\frac{\partial}{\partial s}\right) W_{s}(x, u) d s d u\right),
\end{aligned}
$$


a.e. $x \in \mathbf{R}^{n}$. Here $\alpha(\varepsilon)=\frac{1}{\Gamma\left(\frac{n}{2}\right)} \int_{0}^{\infty} e^{-u} u^{\frac{n}{2}-1} \phi_{\gamma}\left(\frac{\varepsilon^{2}}{4 u}\right) d u, \varepsilon \in(0,1)$.

Since $\phi_{\gamma}(a s)=a^{-i \gamma} \phi_{\gamma}(s), a, s>0$, it follows that, for every $R>0$,

$$
\begin{aligned}
& T_{m_{\gamma}}\left(f_{R}\right)\left(\frac{x}{\sqrt{R}}\right) \\
& =R^{i \gamma} \lim _{\varepsilon \rightarrow 0^{+}}\left(\alpha(\varepsilon \sqrt{R}) f(x)+\int_{|x-u|>\varepsilon \sqrt{R}} f(u) \int_{0}^{\infty} \phi_{\gamma}(s)\left(-\frac{\partial}{\partial s}\right) W_{s}(x, u) d s d u\right) \\
& =R^{i \gamma} T_{m_{\gamma}}(f)(x), \quad \text { a.e. } x \in \mathbf{R}^{n} .
\end{aligned}
$$

As it was proved above, for every $N \in \mathbf{N}$, there exists $R_{N}>0$ such that $\operatorname{supp} f_{R_{N}} \subseteq B\left(\frac{x}{R_{N}}, \rho\left(\frac{x}{R_{N}}\right)\right),|x| \leq N$, and $R_{N} \leq R_{N+1}$. Then, it follows that

$$
\begin{aligned}
T_{m_{\gamma}}(f)(x) & =R_{N}^{-i \gamma} T_{m_{\gamma}}\left(f_{R_{N}} \chi_{B\left(\frac{x}{R_{N}}, \rho\left(\frac{x}{R_{N}}\right)\right)}\right)\left(\frac{x}{\sqrt{R_{N}}}\right) \\
& =R_{N}^{-i \gamma} T_{m_{\gamma}, \ell}\left(f_{R_{N}}\right)\left(\frac{x}{\sqrt{R_{N}}}\right), \quad|x| \leq N, N \in \mathbf{N} .
\end{aligned}
$$

We deduce that,

$$
\begin{aligned}
\int_{B(0, N)}\left|T_{m_{\gamma}}(f)(x)\right|^{p} d x & \leq R_{N}^{n / 2} \int_{\mathbf{R}^{n}}\left|T_{m_{\gamma}, \ell}\left(f_{R_{N}}\right)(x)\right|^{p} d x \\
& \leq C R_{N}^{n / 2} \int_{\mathbf{R}^{n}}\left|f_{R_{N}}(x)\right|^{p} d x \leq C\|f\|_{p}^{p}, \quad N \in \mathbf{N} .
\end{aligned}
$$

Note that $C$ does not depend on $N$.

We conclude that

$$
\left\|T_{m_{\gamma}}(f)\right\|_{p} \leq C\|f\|_{p} .
$$

Also, (15) holds for every $f \in C_{c}^{\infty}\left(\mathbf{R}^{n}\right) \otimes B$. Hence, $T_{m_{\gamma}}$ can be extended to $L^{p}\left(\mathbf{R}^{n}, B\right)$ as a bounded operator from $L^{p}\left(\mathbf{R}^{n}, B\right)$ into itself.

Suppose now that for every $\gamma \in \mathbf{R}$ the operator $T_{m_{\gamma}}^{\mathcal{L}}$ can be extended to $L^{1}\left(\mathbf{R}^{n}, B\right)$ as a bounded operator from $L^{1}\left(\mathbf{R}^{n}, B\right)$ into $L^{1, \infty}\left(\mathbf{R}^{n}, B\right)$. Then, for every $\gamma \in \mathbf{R}$, the operator $T_{m_{\gamma}, \ell}$ can be extended to $L^{1}\left(\mathbf{R}^{n}, B\right)$ as a bounded operator from $L^{1}\left(\mathbf{R}^{n}, B\right)$ into $L^{1, \infty}\left(\mathbf{R}^{n}, B\right)$.

Let $\gamma \in \mathbf{R}$ and $f \in C_{c}^{\infty}\left(\mathbf{R}^{n}\right) \otimes B$. By (14), for every $N \in \mathbf{N}$, there exists $R_{N}>0$ such that

$$
T_{m_{\gamma}}(f)(x)=R_{N}^{-i \gamma} T_{m_{\gamma}, \ell}\left(f_{R_{N}}\right)\left(\frac{x}{\sqrt{R_{N}}}\right), \quad|x| \leq N .
$$

Hence, for every $N \in \mathbf{N}$ and $\lambda>0$, we get

$$
\begin{aligned}
\left|\left\{x \in B(0, N):\left\|T_{m_{\gamma}}(f)(x)\right\|_{B}>\lambda\right\}\right| & \leq R_{N}^{n / 2}\left|\left\{x \in \mathbf{R}^{n}:\left\|T_{m_{\gamma}, \ell}\left(f_{R_{N}}\right)(x)\right\|_{B}>\lambda\right\}\right| \\
& \leq C \frac{R_{N}^{n / 2}}{\lambda} \int_{\mathbf{R}^{n}}\left\|f_{R_{N}}(x)\right\|_{B} d x \\
& \leq C \frac{1}{\lambda} \int_{\mathbf{R}^{n}}\|f(x)\|_{B} d x .
\end{aligned}
$$


Then, by letting $N \rightarrow \infty$, we deduce that

$$
\left|\left\{x \in \mathbf{R}^{n}:\left\|T_{m_{\gamma}}(f)(x)\right\|_{B}>\lambda\right\}\right| \leq C \frac{1}{\lambda} \int_{\mathbf{R}^{n}}\|f(x)\|_{B} d x, \quad \lambda>0 .
$$

Since $C_{c}^{\infty}\left(\mathbf{R}^{n}\right) \otimes B$ is dense in $L^{1}\left(\mathbf{R}^{n}, B\right), T_{m_{\gamma}}$ can be extended to $L^{1}\left(\mathbf{R}^{n}, B\right)$ as a bounded operator from $L^{1}\left(\mathbf{R}^{n}, B\right)$ into $L^{1, \infty}\left(\mathbf{R}^{n}, B\right)$, and by Theorem 4 we conclude that $B$ is UMD.

(i) $\Leftrightarrow\left(\right.$ iv). Let $f \in L^{1}\left(\mathbf{R}^{n}, B\right)$. For every $x \in \mathbf{R}^{n}$ and $0<\varepsilon<\rho(x)$ we can write

$$
\begin{aligned}
& \int_{|x-y|>\varepsilon} f(y) K_{\phi_{\gamma}}^{\mathcal{L}}(x, y) d y-\int_{|x-y|>\varepsilon} f(y) K_{\phi_{\gamma}}(x, y) d y \\
& =\int_{\varepsilon<|x-y|<\rho(x)} f(y)\left(K_{\phi_{\gamma}}^{\mathcal{L}}(x, y)-K_{\phi_{\gamma}}(x, y)\right) d y \\
& \quad+\int_{|x-y| \geq \rho(x)} f(y) K_{\phi_{\gamma}}^{\mathcal{L}}(x, y) d y-\int_{|x-y| \geq \rho(x)} f(y) K_{\phi_{\gamma}}(x, y) d y \\
& =H_{m_{\gamma}, 1}^{\varepsilon}(f)(x)+H_{m_{\gamma}, 2}(f)(x)+H_{m_{\gamma}, 3}(f)(x) .
\end{aligned}
$$

By proceeding as above we have that

$$
\left\|H_{m_{\gamma}, 1}^{\varepsilon}(f)(x)\right\|_{B} \leq C \mathcal{M}\left(\|f\|_{B}\right)(x), \quad 0<\varepsilon<\rho(x), \quad x \in \mathbf{R}^{n},
$$

and

$$
\left\|H_{m_{\gamma}, 2}(f)(x)\right\|_{B} \leq C \mathcal{M}\left(\|f\|_{B}\right)(x), \quad x \in \mathbf{R}^{n} .
$$

Also, since $\left|K_{\phi_{\gamma}}(x, y)\right| \leq C|x-y|^{-n}, x, y \in \mathbf{R}^{n}, x \neq y$, it follows that

$$
\left\|H_{m_{\gamma}, 3}(f)(x)\right\|_{B} \leq C \int_{|x-y| \geq \rho(x)} \frac{\|f(y)\|_{B}}{|x-y|^{n}} d y \leq C \frac{1}{\rho(x)^{n}} \int_{\mathbf{R}^{n}}\|f(y)\|_{B} d y, \quad x \in \mathbf{R}^{n} .
$$

Moreover, if $x \in \mathbf{R}^{n}$ and $\varepsilon \geq \rho(x)$, we have

$$
\begin{aligned}
& \left\|\int_{|x-y|>\varepsilon} f(y) K_{\phi_{\gamma}}^{\mathcal{L}}(x, y) d y-\int_{|x-y|>\varepsilon} f(y) K_{\phi_{\gamma}}(x, y) d y\right\|_{B} \\
& \leq \int_{|x-y| \geq \rho(x)}\|f(y)\|_{B}\left|K_{\phi_{\gamma}}^{\mathcal{L}}(x, y)\right| d y+\int_{|x-y| \geq \rho(x)}\|f(y)\|_{B}\left|K_{\phi_{\gamma}}(x, y)\right| d y \\
& \leq C\left(\mathcal{M}\left(\|f\|_{B}\right)(x)+\frac{1}{\rho(x)^{n}} \int_{\mathbf{R}^{n}}\|f(y)\|_{B} d y\right) .
\end{aligned}
$$

Putting together all the above estimations we deduce that

$$
\left|\mathcal{L}_{*}^{i \gamma}(f)(x)-(-\Delta)_{*}^{i \gamma}(f)(x)\right|<\infty, \quad \text { a.e. } x \in \mathbf{R}^{n} .
$$

Hence, $\mathcal{L}_{*}^{i \gamma}(f)(x)<\infty$, a.e. $x \in \mathbf{R}^{n}$, if and only if, $(-\Delta)_{*}^{i \gamma}(f)(x)<\infty$, a.e. $x \in \mathbf{R}^{n}$, and Theorem 4 implies that (i) $\Leftrightarrow$ (iv).

(i) $\Rightarrow(\mathrm{v})$. By Theorems 1 and 2 , for every $f \in L^{1}\left(\mathbf{R}^{n}\right) \otimes B$, there exists the limit

$$
\lim _{\varepsilon \rightarrow 0^{+}} \mathcal{L}_{\varepsilon}^{i \gamma}(f)(x), \quad \text { a.e. } x \in \mathbf{R}^{n} .
$$

Assume that $B$ is UMD. According to Theorem 4 the maximal operator $(-\Delta)_{*}^{i \gamma}$ is bounded from $L^{1}\left(\mathbf{R}^{n}, B\right)$ into $L^{1, \infty}\left(\mathbf{R}^{n}, B\right)$. By proceeding as above we get

$$
\mathcal{L}_{*}^{i \gamma}(f)(x) \leq C\left(\mathcal{M}\left(\|f\|_{B}\right)(x)+\|f(x)\|_{B}+(-\Delta)_{*}^{i \gamma}(f)(x)\right), \quad \text { a.e. } x \in \mathbf{R}^{n} .
$$


The Maximal Theorem allows us to conclude that $\mathcal{L}_{*}^{i \gamma}$ is bounded from $L^{1}\left(\mathbf{R}^{n}, B\right)$ into $L^{1, \infty}\left(\mathbf{R}^{n}, B\right)$. Then, since $C_{c}^{\infty}\left(\mathbf{R}^{n}\right) \otimes B$ is dense in $L^{1}\left(\mathbf{R}^{n}, B\right)$, for every $f \in$ $L^{1}\left(\mathbf{R}^{n}, B\right)$, there exists the limit

$$
\lim _{\varepsilon \rightarrow 0^{+}} \mathcal{L}_{\varepsilon}^{i \gamma}(f)(x), \quad \text { a.e. } x \in \mathbf{R}^{n} .
$$

(v) $\Rightarrow$ (iv). It is clear.

3.3. Proof of Theorem 2. This proof follows the same way that the one of the $L^{p}$-boundedness of the imaginary power $\mathcal{L}^{i \gamma}$ of $\mathcal{L}, \gamma \in \mathbf{R}^{n}$, when $B$ is a UMD space.

Suppose that $m(\lambda)=\lambda \int_{0}^{\infty} e^{-\lambda t} \phi(t) d t, \lambda \in(0, \infty)$, where $\phi \in L^{\infty}(0, \infty)$. Let $f \in C_{c}^{\infty}\left(\mathbf{R}^{n}\right)$. According to Theorem 1

$$
T_{m}^{\mathcal{L}}(f)(x)=\lim _{\varepsilon \rightarrow 0^{+}}\left(\alpha(\varepsilon) f(x)+\int_{|x-y|>\varepsilon} f(y) K_{\phi}^{\mathcal{L}}(x, y) d y\right), \quad \text { a.e. } x \in \mathbf{R}^{n} .
$$

Also, by (11),

$$
T_{m}(f)(x)=\lim _{\varepsilon \rightarrow 0^{+}}\left(\alpha(\varepsilon) f(x)+\int_{|x-y|>\varepsilon} f(y) K_{\phi}(x, y) d y\right), \quad \text { a.e. } x \in \mathbf{R}^{n} .
$$

Here $\alpha \in L^{\infty}(0, \infty)$.

The operator $T_{m}$ is bounded from $L^{p}\left(\mathbf{R}^{n}\right)$ into itself, for every $1<p<\infty$, and from $L^{1}\left(\mathbf{R}^{n}\right)$ into $L^{1, \infty}\left(\mathbf{R}^{n}\right)$. Moreover $T_{m}$ is a Calderón-Zygmund operator. Hence, the maximal operator $T_{m}^{*}$ defined by

$$
T_{m}^{*}(f)(x)=\sup _{\varepsilon>0}\left|\int_{|x-y|>\varepsilon} f(y) K_{\phi}(x, y) d y\right|
$$

is bounded from $L^{p}\left(\mathbf{R}^{n}\right)$ into itself, for every $1<p<\infty$, and from $L^{1}\left(\mathbf{R}^{n}\right)$ into $L^{1, \infty}\left(\mathbf{R}^{n}\right)$. Also, the same $L^{p}$-boundedness properties are satisfied by the operators

$$
T_{m, \ell}(f)(x)=\lim _{\varepsilon \rightarrow 0}\left(\alpha(\varepsilon) f(x)+\int_{\varepsilon<|x-y|<\rho(x)} f(y) K_{\phi}(x, y) d y\right)
$$

and

$$
T_{m, g}(f)(x)=\int_{|x-y| \geq \rho(x)} f(y) K_{\phi}(x, y) d y .
$$

The difference $T_{m}^{\mathcal{L}}(f)-T_{m, \ell}(f)$ can be written as

$$
\begin{aligned}
& T_{m}^{\mathcal{L}}(f)(x)-T_{m, \ell}(f)(x) \\
& =\int_{|x-y|<\rho(x)}\left(K_{\phi}^{\mathcal{L}}(x, y)-K_{\phi}(x, y)\right) f(y) d y+\int_{|x-y| \geq \rho(x)} K_{\phi}^{\mathcal{L}}(x, y) f(y) d y .
\end{aligned}
$$

By proceeding as in the proof of Theorem 3 we can see that the operator $T_{m}^{\mathcal{L}}-T_{m, \ell}$ is bounded from $L^{p}\left(\mathbf{R}^{n}\right)$ into itself, for every $1<p<\infty$, and from $L^{1}\left(\mathbf{R}^{n}\right)$ into $L^{1, \infty}\left(\mathbf{R}^{n}\right)$.

Hence we conclude that $T_{m}^{\mathcal{L}}$ can be extended to $L^{p}\left(\mathbf{R}^{n}\right), 1<p<\infty$, as a bounded operator from $L^{p}\left(\mathbf{R}^{n}\right)$ into itself, for every $1<p<\infty$, and from $L^{1}\left(\mathbf{R}^{n}\right)$ into $L^{1, \infty}\left(\mathbf{R}^{n}\right)$.

Moreover, we can deduce that the maximal operator

$$
T_{m}^{\mathcal{L}, *}(f)(x)=\sup _{\varepsilon>0}\left|\int_{|x-y|>\varepsilon} K_{\phi}^{\mathcal{L}}(x, y) f(y) d y\right|, \quad x \in \mathbf{R}^{n},
$$


is bounded from $L^{p}\left(\mathbf{R}^{n}\right)$ into itself, for every $1<p<\infty$, and from $L^{1}\left(\mathbf{R}^{n}\right)$ into $L^{1, \infty}\left(\mathbf{R}^{n}\right)$.

Hence, for every $f \in L^{p}\left(\mathbf{R}^{n}\right), 1 \leq p<\infty$, there exists the limit

$$
\lim _{\varepsilon \rightarrow 0^{+}}\left(f(x) \alpha(\varepsilon)+\int_{|x-y|>\varepsilon} f(y) K_{\phi}^{\mathcal{L}}(x, y) d y\right), \quad \text { a.e. } x \in \mathbf{R}^{n},
$$

and, for every $f \in L^{2}\left(\mathbf{R}^{n}\right)$,

$$
T_{m}^{\mathcal{L}}(f)(x)=\lim _{\varepsilon \rightarrow 0^{+}}\left(f(x) \alpha(\varepsilon)+\int_{|x-y|>\varepsilon} f(y) K_{\phi}^{\mathcal{L}}(x, y) d y\right), \quad \text { a.e. } x \in \mathbf{R}^{n} .
$$

We conclude that the operator $T_{m}^{\mathcal{L}}$ can be extended from $L^{2}\left(\mathbf{R}^{n}\right) \cap L^{p}\left(\mathbf{R}^{n}\right)$ to $L^{p}\left(\mathbf{R}^{n}\right)$, $1 \leq p<\infty$, as a bounded operator from $L^{p}\left(\mathbf{R}^{n}\right)$ into itself, for every $1<p<\infty$ and from $L^{1}\left(\mathbf{R}^{n}\right)$ into $L^{1, \infty}\left(\mathbf{R}^{n}\right)$.

\section{Appendix}

In this section we present a pointwise representation of the multiplier $T_{m}$. We establish the properties (11) and (13).

For every $f \in L^{2}\left(\mathbf{R}^{n}\right)$ we have that

$$
T_{m}(f)=\left(m\left(|y|^{2}\right) \hat{f}\right)^{\swarrow}
$$

Let $f \in C_{c}^{\infty}\left(\mathbf{R}^{n}\right)$. We can write

$$
\begin{aligned}
T_{m}(f)(x) & =\frac{1}{(2 \pi)^{n}} \int_{\mathbf{R}^{n}} e^{i x y} m\left(|y|^{2}\right) \hat{f}(y) d y \\
& =\frac{1}{(2 \pi)^{n}} \int_{\mathbf{R}^{n}} e^{i x y} \hat{f}(y)|y|^{2} \int_{0}^{\infty} \phi(t) e^{-t|y|^{2}} d t d y \\
& =\frac{1}{(2 \pi)^{n}} \int_{0}^{\infty} \phi(t) \int_{\mathbf{R}^{n}} e^{i x y}|y|^{2} e^{-t|y|^{2}} \hat{f}(y) d y d t, \quad x \in \mathbf{R}^{n} .
\end{aligned}
$$

The interchange in the order of integration is justified because

$$
\int_{\mathbf{R}^{n}}\left|\hat{f}(y)\left\|\left.y\right|^{2} \int_{0}^{\infty} e^{-t|y|^{2}}|\phi(t)| d t d y \leq\right\| \phi \|_{\infty} \int_{\mathbf{R}^{n}}\right| \hat{f}(y) \mid d y<\infty .
$$

Then,

$$
\begin{aligned}
T_{m}(f)(x) & =\frac{1}{(2 \pi)^{n}} \int_{0}^{\infty} \phi(t) \int_{\mathbf{R}^{n}} e^{i x y} e^{-t|y|^{2}}(\widehat{-\Delta) f}(y) d y d t \\
& =\frac{-1}{(2 \pi)^{n}} \int_{0}^{\infty} \phi(t) \int_{\mathbf{R}^{n}} \Delta f(z) \int_{\mathbf{R}^{n}} e^{-i y(z-x)} e^{-t|y|^{2}} d y d z d t \\
& =-\int_{0}^{\infty} \phi(t) \int_{\mathbf{R}^{n}} \Delta f(z) W_{t}(x, z) d z d t, \quad x \in \mathbf{R}^{n} .
\end{aligned}
$$

We have taken into account that

$$
\int_{\mathbf{R}^{n}} \int_{\mathbf{R}^{n}}|\Delta f(z)| e^{-t|y|^{2}} d z d y<\infty, \quad t>0
$$

and that

$$
\int_{\mathbf{R}^{n}} e^{-i y z} e^{-t|y|^{2}} d y=\left(\frac{\pi}{t}\right)^{\frac{n}{2}} e^{-\frac{|z|^{2}}{4 t}}
$$


Since $\int_{\mathbf{R}^{n}} \Delta f(z) d z=\widehat{\Delta f}(0)=-|y|^{2} \hat{f}(y)_{\mid y=0}=0$, we can write

$$
T_{m}(f)(x)=-\int_{0}^{\infty} \phi(t) \int_{\mathbf{R}^{n}} \Delta f(z)\left(W_{t}(x, z)-\frac{\chi_{(1, \infty)}(t)}{(4 \pi t)^{\frac{n}{2}}}\right) d z d t, \quad x \in \mathbf{R}^{n} .
$$

It is not hard to see that

$$
\left|W_{t}(x, z)-\frac{1}{(4 \pi t)^{\frac{n}{2}}}\right| \leq C \frac{|x-z|^{2}}{t^{\frac{n+2}{2}}}, \quad x, z \in \mathbf{R}^{n} \text { and } t>0 .
$$

Hence it follows that

$$
\begin{aligned}
& \int_{0}^{\infty} \int_{\mathbf{R}^{n}}|\Delta f(z)|\left|W_{t}(x, z)-\frac{\chi_{(1, \infty)}(t)}{(4 \pi t)^{\frac{n}{2}}}\right| d z d t \\
& \leq C\left(\int_{0}^{1} \int_{\mathbf{R}^{n}} W_{t}(x, z) d z d t+\int_{1}^{\infty} \int_{\operatorname{supp} f} \frac{|x-z|^{2}}{t^{\frac{n+2}{2}}} d z d t\right) \leq C\left(1+|x|^{2}\right), \quad x \in \mathbf{R}^{n} .
\end{aligned}
$$

Then,

$$
T_{m}(f)(x)=-\lim _{\varepsilon \rightarrow 0^{+}} \int_{0}^{\infty} \phi(t) \int_{|x-z|>\varepsilon} \Delta f(z)\left(W_{t}(x, z)-\frac{\chi_{(1, \infty)}(t)}{(4 \pi t)^{\frac{n}{2}}}\right) d z d t, \quad x \in \mathbf{R}^{n} .
$$

Let $0<\varepsilon<1$. The Green formula leads to,

$$
\begin{aligned}
& \int_{|x-z|>\varepsilon} \Delta f(z)\left(W_{t}(x, z)-\frac{\chi_{(1, \infty)}(t)}{(4 \pi t)^{\frac{n}{2}}}\right) d z \\
& =\int_{|x-z|>\varepsilon} f(z) \Delta_{z} W_{t}(x, z) d z+\int_{|x-z|=\varepsilon} \partial_{n} f(z)\left(W_{t}(x, z)-\frac{\chi_{(1, \infty)}(t)}{(4 \pi t)^{\frac{n}{2}}}\right) d \sigma(z) \\
& \quad-\int_{|x-z|=\varepsilon} f(z) \partial_{n, z} W_{t}(x, z) d \sigma(z), \quad x \in \mathbf{R}^{n} \text { and } t>0 .
\end{aligned}
$$

Here $\partial_{n}$ represents the derivative in the direction normal exterior to the sphere $S_{\varepsilon}=$ $\left\{z \in \mathbf{R}^{n}:|z-x|=\varepsilon\right\}$.

By using [19, Lemma 2.1] we have that

$$
\begin{aligned}
& \left|\int_{0}^{\infty} \phi(t) \int_{|x-z|=\varepsilon} \partial_{n} f(z)\left(W_{t}(x, z)-\frac{\chi_{(1, \infty)}(t)}{(4 \pi t)^{\frac{n}{2}}}\right) d \sigma(z) d t\right| \\
& \leq C \int_{|x-z|=\varepsilon}\left(\int_{0}^{1} \frac{e^{-\frac{|x-z|^{2}}{4 t}}}{t^{\frac{n}{2}}} d t+\int_{1}^{\infty} \frac{|x-z|^{2}}{t^{\frac{n}{2}+1}} d t\right) d \sigma(z) \\
& \leq C \int_{|x-z|=\varepsilon}\left(\frac{1}{|x-z|^{n-2}}+|x-z|^{2}\right) d \sigma(z) \leq C \varepsilon, \quad x \in \mathbf{R}^{n} .
\end{aligned}
$$

If $n(z)$ denotes a unitary vector in the direction exterior normal in $z \in S_{\varepsilon}$, we obtain

$$
\begin{aligned}
\partial_{n, z} W_{t}(x, z) & =\left\langle\nabla_{z} W_{t}(x, z), n(z)\right\rangle=W_{t}(x, z)\left\langle\frac{x-z}{2 t}, n(z)\right\rangle \\
& =W_{t}(x, z) \frac{|x-z|}{2 t}=\frac{e^{-\frac{\varepsilon^{2}}{4 t}} \varepsilon}{2(4 \pi)^{\frac{n}{2}} t^{\frac{n}{2}+1}}, \quad z \in S_{\varepsilon} .
\end{aligned}
$$


Moreover, $\sigma\left(S_{\varepsilon}\right)=2 \varepsilon^{n-1} \frac{\pi^{\frac{n}{2}}}{\Gamma\left(\frac{n}{2}\right)}$. Then we have that

$$
\begin{aligned}
& \int_{0}^{\infty} \int_{|x-z|=\varepsilon} f(z) \partial_{n, z} W_{t}(x, z) d \sigma(z) \phi(t) d t \\
& =\varepsilon \int_{|x-z|=\varepsilon} f(z) \int_{0}^{\infty} \frac{e^{-\frac{\varepsilon^{2}}{4 t}}}{2(4 \pi)^{\frac{n}{2}}} \frac{\phi(t)}{t^{\frac{n}{2}+1}} d t d \sigma(z) \\
& =\frac{1}{\varepsilon^{n-1} 2 \pi^{\frac{n}{2}}} \int_{|x-z|=\varepsilon} f(z) \int_{0}^{\infty} \phi\left(\frac{\varepsilon^{2}}{4 u}\right) e^{-u} u^{\frac{n}{2}-1} d u d \sigma(z) \\
& =\frac{1}{\varepsilon^{n-1} 2 \pi^{\frac{n}{2}}} \int_{|x-z|=\varepsilon}(f(z)-f(x)) \int_{0}^{\infty} \phi\left(\frac{\varepsilon^{2}}{4 u}\right) e^{-u} u^{\frac{n}{2}-1} d u d \sigma(z)+f(x) \alpha(\varepsilon),
\end{aligned}
$$

$x \in \mathbf{R}^{n}$, where $\alpha(\varepsilon)=\frac{1}{\Gamma\left(\frac{n}{2}\right)} \int_{0}^{\infty} \phi\left(\frac{\varepsilon^{2}}{4 u}\right) e^{-u} u^{\frac{n}{2}-1} d u, 0<\varepsilon<1$.

Since $f$ is a continuous function we get

$$
\lim _{\varepsilon \rightarrow 0^{+}} \frac{1}{\varepsilon^{n-1} 2 \pi^{\frac{n}{2}}} \int_{|x-z|=\varepsilon}(f(z)-f(x)) \int_{0}^{\infty} \phi\left(\frac{\varepsilon^{2}}{4 u}\right) e^{-u} u^{\frac{n}{2}-1} d u d \sigma(z)=0 .
$$

It is clear that $\alpha$ is a bounded function on $(0, \infty)$. Moreover, if there exists $\phi\left(0^{+}\right)=$ $\lim _{t \rightarrow 0^{+}} \phi(t)$, by using the dominated convergence theorem we obtain

$$
\lim _{\varepsilon \rightarrow 0^{+}} \alpha(\varepsilon)=\phi\left(0^{+}\right) \text {. }
$$

Since $\Delta_{z} W_{t}(x, z)=\frac{\partial}{\partial t} W_{t}(x, z), x, z \in \mathbf{R}^{n}$ and $t>0$, the above arguments allow us to establish (11) and (13).

\section{References}

[1] Abu-Falahah, I., P. R. Stinga, and J.L. Torrea: Square functions associated to Schrödinger operators. - Studia Math. 203, 2011, 171-194.

[2] Auscher, P., and B. Ben Ali: Maximal inequalities and Riesz transforms estimates on $L^{p}$ spaces for Schrödinger operators with nonnegative potentials. - Ann. Inst. Fourier (Grenoble) 57:6, 2007, 1975-2013.

[3] Betancor, J. J., J. C. Fariña, E. Harboure, and L. Rodríguez-Mesa. L ${ }^{p}$-boundeness properties of variation operators in the Schrödinger setting. - Rev. Mat. Complut. (to appear), doi:10.1007/s13163-012-0094-y.

[4] Bourgain, J.: Some remarks on Banach spaces in which martingale difference sequences are unconditional. - Ark. Mat. 21:2, 1983, 163-168.

[5] Burkholder. D. L.: A geometrical characterization of Banach spaces in which martingale difference sequences are unconditional. - Ann. Probab. 9:6, 1981, 997-1011.

[6] Burkholder, D. L.: Martingales and Fourier analysis in Banach spaces. - In: Probability and analysis (Varenna, 1985), Lecture Notes in Math. 1206, Springer, Berlin, 1986, 61-108.

[7] Coifman, R. R., and G. Weiss: Transference methods in analysis. - CBMS Reg. Conf. Ser. Math. 31, Amer. Math. Soc., Providence, R.I., 1977.

[8] Dziubański, J., G. Garrigós, T. Martínez, J. L. Torrea, and J. Zienkiewicz: BMO spaces related to Schrödinger operators with potentials satisfying a reverse Hölder inequality. - Math. Z. 249:2, 2005, 329-356. 
[9] García-Cuerva, J., and J. L. Rubio de Francia: Weighted norm inequalities and related topics. - North Holland Publishing Co., Amsterdam, 1985.

[10] Grafakos, L., L. LiU, and D. Yang: Vector valued singular integrals and maximal functions on spaces of homogeneous type. - Math. Scand. 104:2, 2009, 296-310.

[11] Guerre-Delabrière, S.: Some remarks on complex powers of $(-\Delta)$ and UMD spaces. Illinois J. Math. 35:3, 1991, 401-407.

[12] Hytönen, T.P.: Aspects of probabilistic Littlewood-Paley theory in Banach spaces. - In: Banach spaces and their applications in analysis, Walter de Gruyter, Berlin, 2007, 343-355.

[13] Hytönen, T. P.: Littlewood-Paley-Stein theory for semigroups in UMD spaces. - Rev. Mat. Iberoam. 23:3, 2007, 973-1009.

[14] Martínez, T., J. L. Torrea, and Q. Xu: Vector-valued Littlewood-Paley-Stein theory for semigroups. - Adv. Math. 203:2, 2006, 430-475.

[15] Rubio de Francia, J.L., F. Ruiz, and J. L. Torrea: Calderón-Zygmund theory for operator-valued kernels. - Adv. Math. 62:1, 1986, 7-48.

[16] Shen, Z.W.: $L^{p}$ estimates for Schrödinger operators with certain potentials. - Ann. Inst. Fourier (Grenoble) 45:2, 1995, 513-546.

[17] Stein, E. M.: Topics in harmonic analysis related to the Littlewood-Paley theory. - Ann. of Math. Stud. 63, Princeton Univ. Press, Princeton, N.J., 1970.

[18] Stein, E. M., and G. Weiss: Introduction to Fourier analysis on Euclidean spaces. - Princeton Math. Ser. 32, Princeton Univ. Press, Princeton, N.J., 1971.

[19] Stempak, K., and J. L. Torrea: Poisson integrals and Riesz transforms for Hermite function expansions with weights. - J. Funct. Anal. 202:2, 2003, 443-472.

[20] XU, Q.: Littlewood-Paley theory for functions with values in uniformly convex spaces. - J. Reine Angew. Math. 504, 1998, 195-226.

[21] Zimmermann, F.: On vector valued Fourier multiplier theorems. - Studia Math. 93, 1989, $201-222$. 